\title{
The mass function of nearby black hole candidates
}

\author{
L. I. Caramete ${ }^{1,2, \star}$ and P. L. Biermann ${ }^{1,3,4,5,6}$
}

\author{
1 Max Planck Institute for Radio Astronomy, Auf dem Hügel 69, 53121 Bonn, Germany \\ 2 Institute for Space Sciences, PO Box MG-23, Ro 077125 Bucharest-Magurele, Romania \\ e-mail: lcaramete@venus.nipne.ro \\ Department of Physics and Astronomy, University of Bonn, Endenicher Allee 11-13, Bonn, Germany \\ ${ }^{4}$ Department of Physics and Astronomy, University of Alabama, Tuscaloosa, Alabama 35487, USA \\ 5 Department of Physics, University of Alabama at Huntsville, 301 Sparkman Drive Huntsville, AL 35899, USA \\ ${ }^{6}$ Karlsruhe Institute of Technology, PO Box 3640, 76021 Karlsruhe, Germany
}

Received 19 August 2009 / Accepted 13 July 2010

\section{ABSTRACT}

\begin{abstract}
Context. The mass function of supermassive black holes in our cosmic neighborhood is required to understand the statistics of their activity and consequently the origin of ultra high energy particles.

Aims. We determine a mass function of supermassive black hole candidates from the entire sky except for the Galactic plane.

Methods. Using the 2MASS catalogue as a starting point, and the well-established correlation between black hole mass and the bulge of old population of stars, we derive a list of nearby black hole candidates within the redshift range $z<0.025$, then perform an additional selection based on the Hubble type. We present our resulting catalogue elsewhere. The final list of black hole candidates above a mass of $M_{\mathrm{BH}}>3 \times 10^{6} M_{\odot}$ has 5829 entries. We perform a Hubble-type correction to account for selection effects, which reduces this number to 2919 black hole candidates. Here we use this catalogue to derive the black-hole mass function. We also correct for volume, so that this mass function is a volume-limited distribution to redshift 0.025 .

Results. The differential mass function of nearby black hole candidates is a curved function, with a straight simple power-law of index -3 above $10^{8} M_{\odot}$ that becomes progressively flatter towards lower masses, turns off towards a gap below $3 \times 10^{6} M_{\odot}$, and then extends into the range where nuclear star clusters replace black holes. The shape of this mass function can be explained in a simple merger picture. Integrating this mass function over the redshift range for which it has been derived, infers a total number of black holes with $z<0.025$, and $M_{\mathrm{BH}}>10^{7} M_{\odot}$ of about $2.4 \times 10^{4}$, or, if we average uniformly, 0.6 for every square degree on the sky.
\end{abstract}

Key words. black hole physics - galaxies: general - acceleration of particles

\section{Introduction}

In the quest to identify the origin of ultra high energy cosmic-ray particles, many proposed mechanisms use the power of supermassive black holes, obtained either from accretion (e.g., Falcke \& Biermann 1995), from spin-down (e.g., Blandford \& Znajek 1977), or in some other way (e.g., Biermann \& Frampton 2006). It is not a priori clear, what is the most effective constraining argument to use when trying to identify which small fraction of all supermassive black holes produce ultra high energy particles. As long as the mechanism is unclear, we need to consider all plausible supermassive black holes. Therefore, it is important to obtain the mass function of black holes in order to estimate the number of black holes to within the sphere from where ultra high energy particles might come. Since various other groups have also derived the mass function of black holes, using different methods, we have a good way of checking our work.

Supermassive black hole characteristics are strongly correlated with the properties of their host galaxies, and we use this correlation to obtain abundant statistics, but also to control the quality of our data set. We wish to address a number of questions related to the super-massive black holes in the universe: a) it appears that super-massive black holes have a low cut-off

* Member of the International Max Planck Research School (IMPRS) for Astronomy and Astrophysics at the Universities of Bonn and Cologne. in their mass distribution, which it is important to verify and whose existence we should try to understand; it also appears that the black holes mass function has an upper limit, and we need to understand whether this is a statistical fluke or has a physical meaning; b) what is the slope of the mass function? This will surely constrain our insight into how black holes grow; c) what is the energy input into the universe during the growth? This is an additional constraint; d) what is the number of black holes? This will constrain any search for directional correlations with ultra high energy cosmic-ray events.

\section{Derivation}

Super-massive black holes are common at the centers of galaxies, and their mass is correlated with the properties of the surrounding galaxy, for instance the velocity dispersion of the galactic stars, and the mass of stars in the spheroidal distribution of the old stellar population.

Since the old stellar population has a spectrum peaking near 2 microns $\left(v S_{v}\right)$ (van der Wel et al. 2006), and it is the properties of this population that correlates with those of the black holes, we use the 2MASS catalogue as our starting point (Skrutskie et al. 2006): we focus on early Hubble-type galaxies (Caramete \& Biermann, in prep.) and use the black-hole mass-spheroid correlation (e.g., Faber et al. 1997; Magorrian et al. 1998; Wang \& Biermann 1998; Silk \& Rees 1998; Häring \& Rix 2004; 
Tremaine et al. 2002; Aller \& Richstone 2007) to derive the black hole mass function. We select down to $0.03 \mathrm{Jy}$ at $2 \mathrm{mi}-$ crons, which is far brighter than the 2MASS catalogue completeness limit.

The procedure is as follows:

Step 1: We select first the 2 micron flux-density-limited sample of 10284 . We use all the distance corrections available for this sample.

Step 2: We limit the sample to well determined Hubble types of only early Hubble type, obtaining a sample of size 5894.

Step 3: We attempt to eliminate all galaxies for which we do not have an equivalent method to find a black hole of a given mass for all Hubble types. This reduce the sample by another factor of two to 2928.

For this sample of 2928, we show that the luminosity function exhibits the classical Press-Schechter function (Schechter 1976), a powerlaw with an exponential cutoff. This agrees with previous work in the literature, but uses a different approach, suitable for larger numbers of black holes.

Step 4: We derive the semi-final $\mathrm{BH}$ candidate mass function, extending apparently down to about $10^{5}$ solar masses.

We now describe our procedure in more detail.

The mass of the central black hole is proportional to the flux density of the stellar emission at 2 micron and also proportional to the luminosity distance squared. We need to determine the proportionality constant. This constant differs however for different Hubble types of galaxies, and we have to determine the constant separately, and then check for self-consistency. Since the spheroidal stellar population is far more compact in size for later Hubble types, these differences can be large. Because the estimate of the black hole masses uses the redshift, which is a poor indicator of the true distance at distances smaller than that of the Virgo cluster, we use the available distances provided by the work of B. F. Madore and I. P. Steer ${ }^{1}$ who compiled a database of 3065 accurate, contemporary distances to 1073 galaxies with modest recessional velocities (that is, less than $1 / 8 c$ ) published almost exclusively between 1990 and 2006. From this, we match 429 distances to those in the catalog of massive black hole, and for the rest of distances smaller than that of the Virgo cluster we used distances with respect to only the Virgo infall provided by NED.

We use known black hole masses to calibrate the various early Hubble types: this control sample comprises 58 black holes selected from the recent literature where we use only galaxies earlier than type $\mathrm{Sc}$, including type $\mathrm{Sb}$. These fits provide an error bar of \pm 0.4 dex, slightly worse than the \pm 0.3 dex error given by more sophisticated fits using a much better but also much smaller data set (Aller \& Richstone 2007). These corrections affect a large fraction of the candidate black holes. The final list above $3 \times 10^{6} M_{\odot}$ comprises 5829 black hole candidates. The main error is \pm 0.4 dex, which affects most of the binning of the sample.

In Table 1, we show how many galaxies of various kinds are excluded, and it becomes obvious that from these statistics at worst we have a factor of less than 2 error, if all excluded galaxies of no known Hubble type are indeed either E or S0.

We show how the correction factor depends on the Hubble type relative to the typical value for elliptical galaxies in Fig. 1.

\footnotetext{
${ }^{1}$ http://nedwww.ipac. caltech. edu/level5/NED1D/intro. html
}

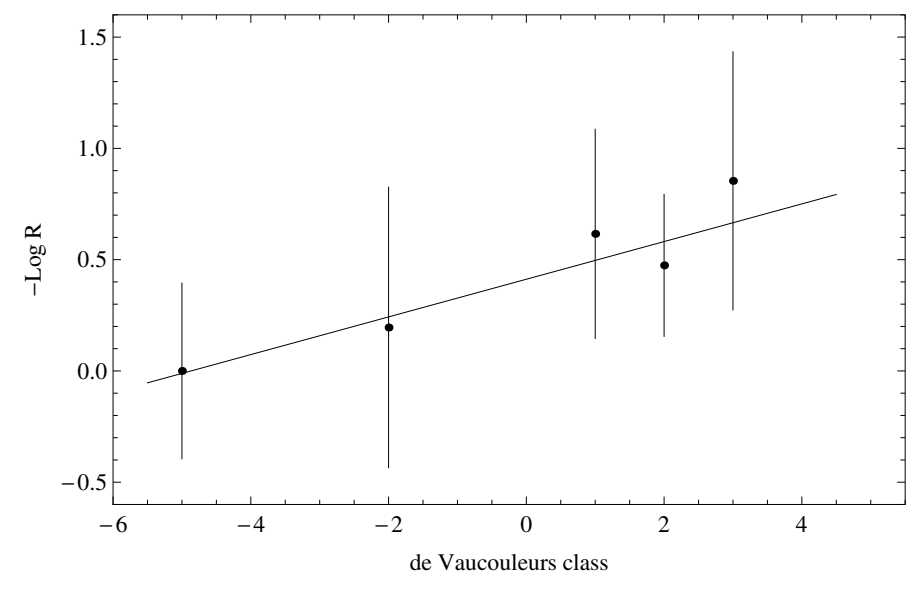

Fig. 1. This is the correction factor required to obtain the black hole mass from the 2 micron emission of the old stellar population, as a function of Hubble type, relative to elliptical galaxies.

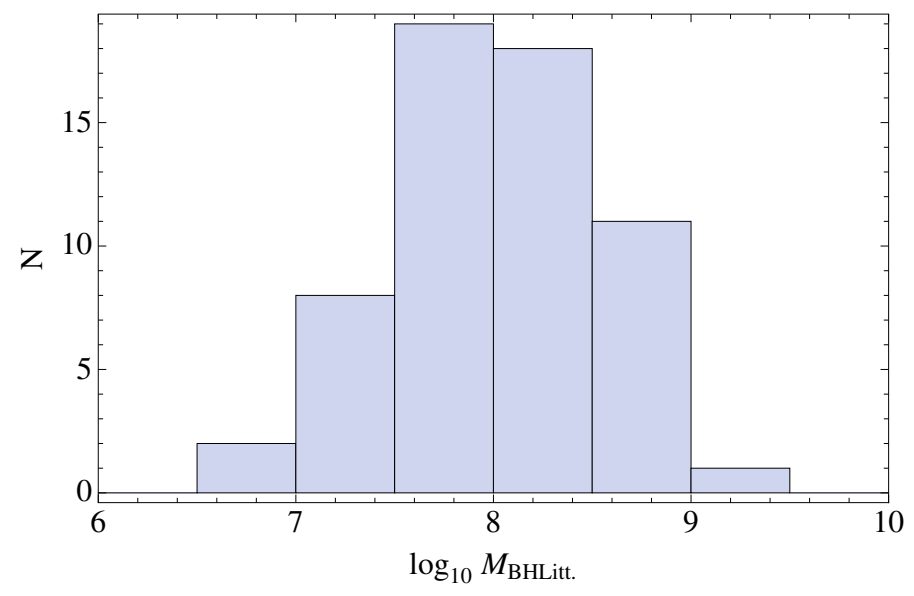

Fig. 2. Histogram of the calculated black hole masses for the sample of black holes obtained from the literature.

Table 1. The number of galaxies of different type for each selection.

\begin{tabular}{lccc}
\hline \hline Type & 5894 selection & 2928 selection & Rejected list \\
\hline E & 783 & 765 & - \\
$\mathrm{S} 0$ & 2626 & 1771 & - \\
$\mathrm{Sa}$ & 879 & 184 & - \\
$\mathrm{Sb}$ & 1052 & 128 & - \\
$\mathrm{Sab}$ & 554 & 80 & - \\
\hline $\mathrm{Sc}$ & - & - & 1941 \\
$\mathrm{Irr}$ & - & - & 14 \\
$\mathrm{Sy}$ & - & - & 128 \\
No type & - & - & 867 \\
Sbrst & - & - & 421 \\
Unknown type & - & - & 1019 \\
\hline
\end{tabular}

In Fig. 2, we show that we have galaxies with black hole masses over the entire range of $10^{6} M_{\odot}$ to $10^{9} M_{\odot}$ as calibrators.

We derive a mass function by going to the minimum mass at each redshift, for which the list is complete to the chosen flux density in the 2MASS catalogue, starting with 0.025 and going down, always correcting for smaller volume. In our adopted procedure, we obtain the number of black holes in several bins above the minimum mass that we can find for both spirals and ellipticals, at redshift $z=0.025$. We then go down in redshift, correcting for different accessible volumes for a complete sample. Figure 3 illustrates the procedure. 
L. I. Caramete and P. L. Biermann: The mass function of nearby black hole candidates

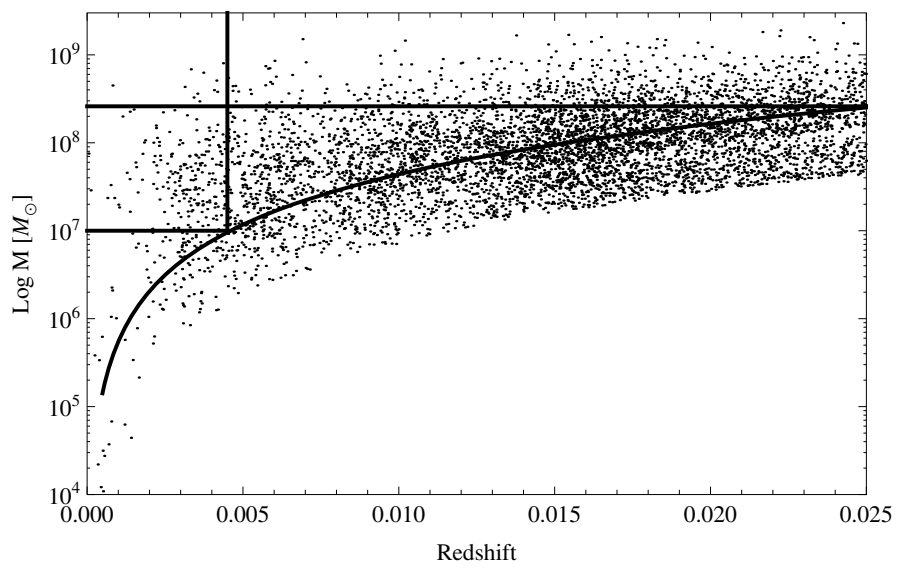

Fig. 3. Plot of mass over redshift for the massive black hole catalog with the selection curve for elliptical galaxies. This also shows the stepwise selection procedure with the two most extreme cases of the lowest mass and the highest redshift.

This diagram of black hole mass versus redshift shows clearly that near the flux density limit, at any redshift, we have only spirals since for a given redshift and given flux density, elliptical galaxies have an inferred black hole mass a factor of up to 5 higher than spirals, Sb spirals having the greatest factor. To eliminate this bias, we divided the entire sample again such that we have a black hole mass limit at each redshift, which allows both spirals and ellipticals to enter the sample. This procedure eliminates many, but not all, of the late type spirals from our final sample for determining the mass function. The structures seen in this diagram illustrate the bias introduced by the correction factor when deriving black hole masses from flux-density-limited data. This reduces the fraction of spirals in the final "Hubbleselected" sample to about twenty percent, so greatly reducing the error contribution from the Hubble-type correction shown above.

We therefore follow the curved line in Fig. 3, determine the number in that mass bin, and calculate the ratio relative to volume; hence we continue to add bins at lower mass until we reach the smallest bin that we wish to consider. This procedure ends at redshift $z=0.0045$ for $M>10^{7} M_{\odot}$, and at $z=0.0024$ for $M>3 \times 10^{6} M_{\odot}$.

We now present our luminosity and mass functions obtained using this final selection of galaxies and their black hole candidates.

The luminosity functions (Fig. 4) agree with previous results in the literature, as we discuss below; they are all well fitted with a Press-Schechter law (1974), a power law with an exponential cutoff. Since our completeness criterion differ from that of most others, we note that our luminosity functions are similar. Since we selected far above the completeness limit of the 2MASS sky survey, and aim to select a galaxy sample that consists of almost 80 percent elliptical and S0-galaxies, our procedure of selection may be more complete for the task at hand, i.e., to determine the black hole mass function, independent of any other property of the black hole or the activity of its surroundings.

The errors included here include an estimate from the binning error induced by uncertain distances, and of course, also Poisson statistics, which are strongly relevant only to the highest mass bin where we have 25 galaxies with their presumed black holes. We consciously ignored all super-massive black holes in late Hubble type galaxies, which implies that our numbers are underestimates. Correcting for the Galactic plane using a

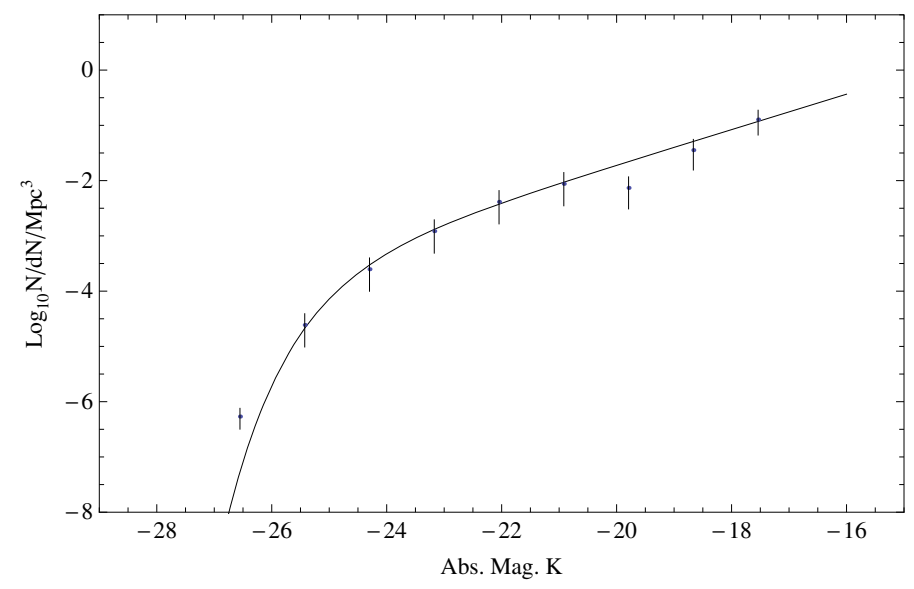

Fig. 4. Integral luminosity function corrected for Hubble-type sampling, 2928 objects selected in $K$-band, the parameters of the Schechter function fit are $M_{*}=-24.3, \phi_{*}=0.0008$ and $\alpha=-1.8$.

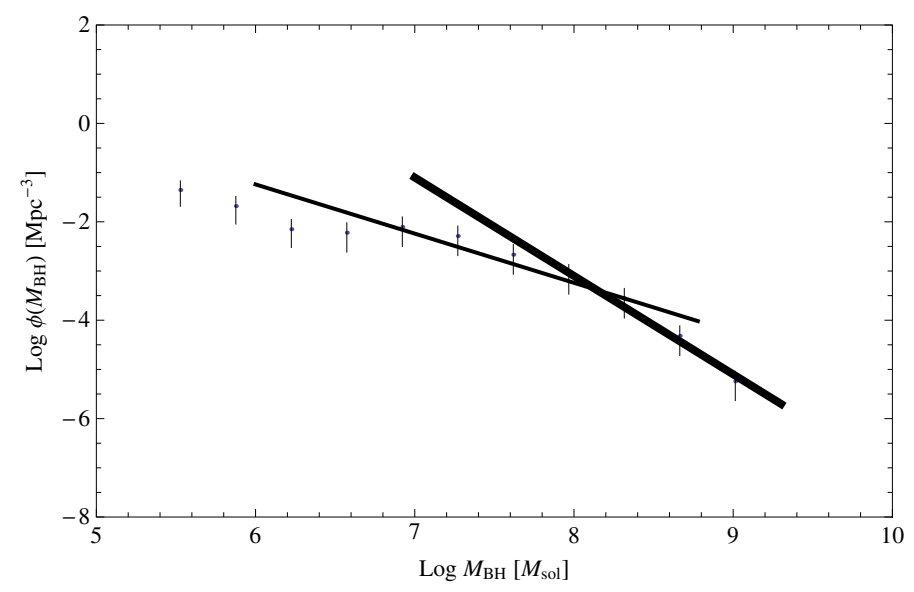

Fig. 5. Integral mass function corrected for Hubble type sampling, 2928 objects, the slope of the lines is: thick line -2.0 fitting $>10^{8} M_{\odot}$, and thin line -1.0 fitting between $10^{7} M_{\odot}$ and $10^{8} M_{\odot}$.

histogram of numbers per latitude bin (see Caramete \& Biermann, in prep.) increases our number by 12.5 percent. We show the result in Fig. 5, with error bars and fits through the data.

Since it is unlikely that all these galaxies actually include a central black hole, we cut the distribution near $3 \times 10^{6} M_{\odot}$, the mass of our Galactic center black hole; we obtain, including the correction for the missing Galactic plane (12.5 percent)

$\phi\left(>M_{\mathrm{BH}}\right)=6 \times 10^{-3 \pm 0.4}\left(\frac{M_{\mathrm{BH}, \mathrm{min}}}{10^{7} M_{\odot}}\right)^{-1.0} \mathrm{Mpc}^{-3}$,

between about $10^{7} M_{\odot}$ and about $10^{8} M_{\odot}$, and

$\phi\left(>M_{\mathrm{BH}}\right)=9 \times 10^{-4 \pm 0.4}\left(\frac{M_{\mathrm{BH}, \mathrm{min}}}{10^{8} M_{\odot}}\right)^{-2.0} \mathrm{Mpc}^{-3}$,

above about $10^{8} M_{\odot}$. These two laws are reasonably good fits, and match near $1.5 \times 10^{8} M_{\odot}$. We imposed the exponents pragmatically, since they can be determined from the data only with large error bars, strongly depending on which bins are included. Simple power laws are adequate.

Between a nominal $10^{6} M_{\odot}$ and $3 \times 10^{6} M_{\odot}$, we have only 6 candidates, between $3 \times 10^{6} M_{\odot}$ and $10^{7} M_{\odot}$ we have 46 candidates, and between $10^{7} M_{\odot}$ and $3 \times 10^{7} M_{\odot}$ we have 185 . In 


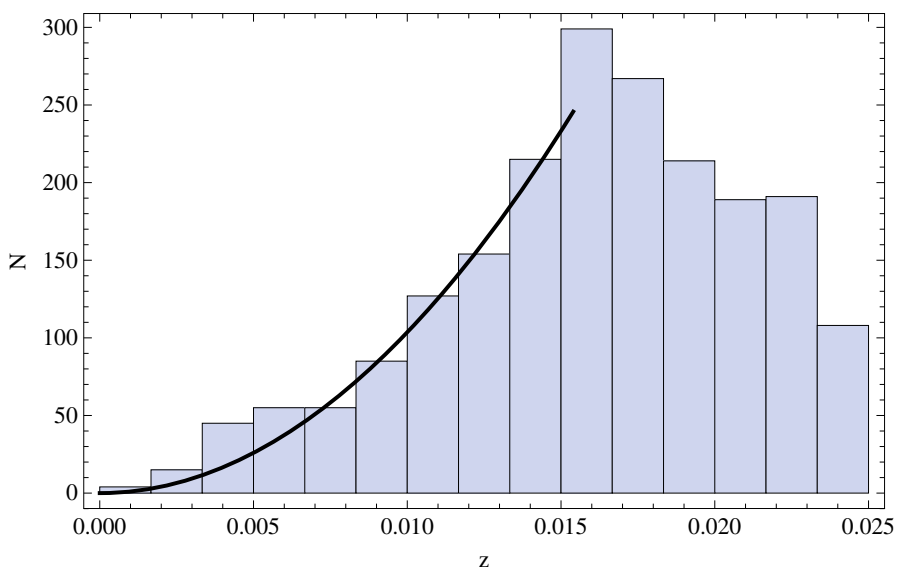

Fig. 6. Histogram of the black hole candidates, corrected for Hubble type sampling, Hubble type correction, cut in BH mass at $1 \times 10^{8} M_{\odot}$, 2023 objects, with a $z^{2}$ fit valid for a homogeneous universe.

the mass range between $10^{6} M_{\odot}$ and $10^{7} M_{\odot}$, there is increasing uncertainty at lower masses, about whether we really detect a black hole, and not just a nuclear star cluster.

Comparing this and various other fits that we tried, we summarize these attempts including error bars from the original calibration to various Hubble types; however, after our correction for Hubble type selection there are few Sb spirals left in the sample (128). Comparing a fit to both the ellipticals and S0s and the entire sample, after applying a low mass cut, with that without any cut provides an estimate of the error in the absolute normalization of about 0.25 in the $\log$; since there are systematics in allocating Hubble types, and in either including or excluding galaxies, we increase our estimate of the error by about $\sqrt{3}$ to a value of 0.4 in the $\log$. We estimated the errors in the absolute scaling, and the exponent from the fit. A lowering of the low mass cutoff by a factor of 3 from $10^{7} M_{\odot}$ to $3 \times 10^{6} M_{\odot}$ corresponds to a negligible increase in the integral number; the integral mass function is nearly flat in that range, which of course implies that the differential mass function in this interval is consistent with zero. A final test was perforemed in which the mass-redshift diagram was cut even more severely to check for edge effects in the sampling, the black hole density increasing marginally, less than 0.2 in the $\log$.

Since any evolution in the black hole mass function is expected to vary approximately with $(1+z)^{4 \pm 1}$, up to a redshift of 0.025 any evolutionary effects ought to be negligible. On the other hand, it is unclear that we have reached the distance range over which the universe is already perfectly homogeneous, a distance possibly larger than 500 Mpc (Rudnick et al. 2007; Kashlinsky et al. 2008); there is no reliable statistical evidence that galaxies in the universe alter their properties dramatically between $100 \mathrm{Mpc}$ and $500 \mathrm{Mpc}$ or even across a widwr volume of the local universe.

In Fig. 6, we show our numbers of black holes above $10^{8} M_{\odot}$ as a function of redshift and the fit to redshifts of a little above 0.015 , where our procedure should be fairly complete for such a mass limit. A homogeneous universe fit is amazingly adequate, but the data also clearly demonstrate that near the redshift limit we begin to miss galaxies, here about 15 percent of the total.

\section{Comparisons}

There have been a large number of studies of black hole mass functions, and their energy input, and here we compare with these papers.

\subsection{Numbers of black holes}

We obviously cannot be certain that all these black hole candidates are really black holes: this question was thoroughly investigated by Greene et al. (2008), who could not find a large number of black holes below $10^{6} M_{\odot}$. We remain unable to state the extent to which this amounts to a selection effect. On the other hand, it was shown by Côté et al. (2006) that the correlations between galaxy parameters and super-massive black hole mass apparently seamlessly merge into a correlation with nuclear star clusters. Nuclear star clusters can in turn become super-massive stars, which collapse to form black holes (Sanders 1970; Portegies Zwart et al. 2004). This may in turn also explain the mass of the black holes near the transition.

The mass function is probably most accurately determined near a mass of $10^{8} M_{\odot}$, where we get a density of $9 \times$ $10^{-4 \pm 0.40} \mathrm{Mpc}^{-3}$. At that mass the density agrees, within the error bars, with both Lauer et al. (2007), who show a possible range from $2 \times 10^{-4} \mathrm{Mpc}^{-3}$ to $5 \times 10^{-3} \mathrm{Mpc}^{-3}$, and Benson et al. (2007), who show a number near $2 \times 10^{-3} \mathrm{Mpc}^{-3}$ (after correcting for the Hubble constant).

Greene \& Ho (2007a) determined the black hole mass function, with particular emphasis on low masses, selecting galaxies on the basis of their activity. First, their numbers show that this distribution peaks near $10^{7} M_{\odot}$, confirming that it may be reasonable to apply a cut at this mass. Second, they confirm the slope which we obtain. However, their total numbers are very much lower - by a factor of about 5000 - than in Lauer et al. (2007); Benson et al. (2007), and our results because of their very different selection procedure, which was based on the activity of black holes.

We conclude here, that our statistics are consistent with those of earlier studies.

\subsection{Energy input from black holes}

As black holes grow, they accrete from an emitting accretion disk, whose luminosity depends on the growth rate, and their final mass is therefore a direct measure of all growth (Lüst 1952; Shakura \& Sunyaev 1973; Novikov \& Thorne 1973). In a similar way, all reliably studied active galactic nuclei have radio emission (e.g., Nagar et al. 2005), which we interpret as originating in a relativistic jet (e.g., Falcke \& Biermann 1995); however, in this case also, all energy dissipated and emitted by the jet is a measure of growth. On the other hand, mergers of black holes (e.g., Gergely \& Biermann 2009), are more likely to emit abundant gravitational waves, which have been invisible so far in the normal universe. To study this, we need to obtain the total growth in black holes, hence their total mass.

If we integrate from $10^{7} M_{\odot}$, the total mass density in black holes is given by

$$
2.8 \times 10^{5 \pm 0.4} M_{\odot} \mathrm{Mpc}^{-3}
$$

which is barely dependent on where we cut the distribution, since at low masses the integral function goes flat, and at high masses the distribution becomes steep.

This compares, for instance with the number obtained by Kronberg et al. (2001), which has been derived from radio emission signatures, and so depends on the efficiency of the conversion process. These authors obtain a lower limit of

$$
\gtrsim 2.2 \times 10^{5} \epsilon_{0.1}^{-1} M_{\odot} \mathrm{Mpc}^{-3},
$$


where $\epsilon_{0.1}$ is the efficiency in units of ten percent. Kronberg et al. (2001) derived their numbers from a selection of higher mass black holes, and provide a lower limit. One could argue that all power output by black holes in the mass range between $10^{6} \mathrm{M}_{\odot}$ and $10^{7} M_{\odot}$ mostly influences the host galaxy, and is less relevant to the intergalactic medium; it could, for instance, mostly contribute to driving a galactic wind (see Everett et al. 2008; Breitschwerdt 2008). We ignored this contribution above.

We can simply convert the units to derive the mass density derived to be

$1.9 \times 10^{-35 \pm 0.40} \mathrm{~g} \mathrm{~cm}^{-3}$,

which corresponds to a cosmological density of (assuming a Hubble constant of $70.1 \mathrm{~km} \mathrm{~s}^{-1} \mathrm{Mpc}^{-1}$ and a critical density of $9.77 \times 10^{-30}$ Komatsu et al. 2009)

$\Omega_{\mathrm{BH}}=2 \times 10^{-6 \pm 0.40}$,

where the error bar in the first factor corresponds to a range of $0.8 \times 10^{-6}$ to $5 \times 10^{-6}$.

Assuming that growth of black holes is mostly via baryonic accretion (Shakura \& Sunyaev 1973; Novikov \& Thorne 1973), the energy input into intergalactic space from the production of all these black holes is given by

$1.9 \times 10^{-15 \pm 0.40} \epsilon_{0.1} \mathrm{erg} \mathrm{cm}^{-3}$,

which is by a factor of order $4.5 \times 10^{-3}-$ excluding the uncertainties - far below that due to the microwave background (at $4.2 \times 10^{-13} \mathrm{erg} \mathrm{cm}^{-3}$ ); this number is an average over all space, derived from the local universe to redshift 0.025 .

We need to compare this estimate with the energy density visible directly in the electromagnetic background, with the energy density inferred to be there in the form of turbulent motion, heat, magnetic fields, and energetic particles, and comprehensive simulations of all these processes.

The electromagnetic radiation background in other wavelengths is approximately (compilation by Kneiske 2009, priv. comm.) at FIR and optical frequencies lower than the microwave background by $5 \times 10^{-3}$, in X-rays lower by $5 \times 10^{-5}$, and yet lower at higher frequencies to a factor of $1.5 \times 10^{-6}$ for $\gamma$-rays. The energy input from the growth of black holes, even at only 10 percent efficiency as used above, exceeds all other energy densities, and could be marginally consistent with the FIR and optical background.

The energy input has also been estimated by Gopal-Krishna \& Wiita (2001), and Enßlin et al. (1998). Enßlin et al. obtain (2.5-4.0) $\times 10^{-16} \mathrm{erg} / \mathrm{cm}^{3}$, while Gopal-Krishna et al. derive $2 \times 10^{-15} \mathrm{erg} / \mathrm{cm}^{3}$ in filaments, similar to what we obtain. We conclude that our numbers are consistent with earlier estimates, which were derived differently.

We should also compare this with the numbers inferred from simulating the turbulent dynamo in the intergalactic medium (Ryu et al. 2008); Ryu (2009), private communication. In this case, we need to compare our numbers with the energy directed into thermal motions and magnetic fields. Ryu obtained a magnetic field energy density of $2 \times 10^{-17} \mathrm{erg} / \mathrm{cm}^{3}$ averaged over the universe at zero redshift. In filaments they determined a magnetic field energy density of $1 \times 10^{-16} \mathrm{erg} / \mathrm{cm}^{3}$, on average a thermal energy density $3 \times 10^{-16} \mathrm{erg} / \mathrm{cm}^{3}$, and in kinetic energy a density $1 \times 10^{-15} \mathrm{erg} / \mathrm{cm}^{3}$. All this energy in these simulations originates in the gravitational wells of the dark-matter large-scale structure, including the galactic halos. Any need to inject far more energy from the activity and growth of central black holes could present a serious problem. The energy input from active galactic nuclei into magnetic fields might be far less than 10 percent efficient; to satisfy observational constraints, we then have the condition

$1 \times 10^{-2 \pm 0.4}>\epsilon_{\mathrm{B}}$

where $\epsilon_{\mathrm{B}}$ is here the efficiency with which we can channel energy from black hole growth into overall magnetic field. We might satisfy this by arguing that far less than 10 percent of all black holes ever become radio galaxies, or alternatively that all supermassive black holes are radio galaxies for much less than 10 percent of their time, and much less than 1 percent of their accretion energy is channelled into magnetic fields (we need a factor of $2 \times 10^{-2}$ ). This may then be consistent with the reasoning of Kronberg et al. (2001), who consider only the manifest energy blown into the intergalactic medium by radio galaxies, traceable through their nonthermal radio emission, to derive numbers for the black hole density.

There are several ways out of this possible dilemma, an obvious answer being that the fraction of energy going into various channels, i.e., electromagnetic, kinetic and magnetic fields remains unclear. A last resort is to note that much of the energy emitted during the growth of black holes via mergers could go into gravitational waves.

\subsection{Extrapolation to lower masses?}

We must recall that we cannot be absolutely sure that the low mass cutoff is at $10^{7} M_{\odot}$, because it might be on a stellar scale (e.g., Clark et al. 2002; Irwin et al. 2009). There are very few confirmed black hole candidates with a mass that significantly exceeds $10 M_{\odot}$, and is below $10^{5} M_{\odot}$ (see van Kerkwijk et al. 1996; Trudolyubov \& Priedhorsky 2004; Gebhardt et al. 2005; Patruno et al. 2006; Maccarone \& Koerding 2006; Orosz et al. 2007; Ulvestad et al. 2007; Casella et al. 2008; Silverman \& Filippenko 2008; Abubekerov et al. 2009; Farrell et al. 2009). One of the most convincing cases for an intermediate mass black hole is the candidate in the cluster G1 within the galaxy M 31 also known as Andromeda.

Since the integral mass function flattens out just near the mass, where there may be very few black holes anymore, there may be little mass below $3 \times 10^{6} M_{\odot}$. The mass to there is only $2.8 \times 10^{5 \pm 0.4} M_{\odot} \mathrm{Mpc}^{-3}$. This is far below the numbers for stellar mass black holes, according to some estimates (Agol \& Kamionkowski 2002). We note that the total mass in stellar size black holes inside our Galaxy is estimated to be of the order of a few $10^{9} M_{\odot}$ to which our law clearly does not connect. It is then reassuring that there is little evidence for black holes between 20 and $10^{5} M_{\odot}$ (Barth et al. 2004, 2005, 2008a,b, 2009; Greene et al. 2006; Greene \& Ho 2007a,b; Greene et al. 2008), and there appears to be many black holes between $10^{6} M_{\odot}$ and $10^{7} M_{\odot}$, but probably not enough to fill the distribution. These stellar black holes increase a factor to the cosmological density of black holes overall.

The finding of few black holes in this gap region is consistent with many other arguments (Körding et al. 2002), implying that there may be no or very few intermediate mass black holes. On the other hand, since the accretion statistics of any hypothetical intermediate-mass black holes are unknown, the observed distribution of candidate sources (Körding et al. 2002) might just be compatible with our statistics. If we were to require that this energy density stays at the level of the $10^{-5}$ fluctuations in the microwave background, then $M_{\mathrm{BH} \text {,min }}$ would have to be beyond 
the upper end of the distribution. Whatever the energy transmitted during the growth of black holes to the observed distribution, it cannot have much effect on the low wave-number fluctuations in the microwave background.

To spin this out, one could imagine that black holes grow initially fast by feeding on dark matter (Munyaneza \& Biermann 2006, 2005) and then continue to grow mostly by mergers; in this scheme, most of the black hole feeding would have come from dark matter. The gravitational waves emitted by all these growth events and mergers might obey the equation of state for dark energy (see Marochnik et al. 2008). It does not appear convincing that first all dark matter has to be black holes (see Abramowicz et al. 2009), and second that all dark energy originate in gravitational waves. At present, this extreme situation does not appear to be a viable solution.

\section{The slope of the mass function}

The shape of the mass function constrains the growth of the distribution. A simple power law at high masses suggests a selfsimilar process.

Silk \& Takahashi (1979) illustrated how it is possible to estimate the building of a mass function from a repeated merger process, building on yet earlier work. We now consider whether their approach can also explain the slope that we find here.

We assume that all black holes grow by merging with other black holes and that baryonic accretion is just a multiplying factor, which is equivalent to perhaps always adding a factor of 1.5 in mass. It may well represent a narrow distribution of added fractional mass.

When writing the coalescence rate as $\sim(\text { Mass })^{\lambda}$, they find that the resulting mass function in its power law form becomes $\sim m^{-3 \lambda / 2}$. In our context, this implies that $\lambda=2.0$. This then says that the product of cross-section, typical velocity, and sticking probability also follow this combined dependence on mass. In Gergely \& Biermann (2009) we argued that the cross section increases as (Mass) ${ }^{1 / 2}$. On the other hand, small systems such as the Local Group, have a lower typical velocity of order $200 \mathrm{~km} \mathrm{~s}^{-1}$ than larger systems such as clusters of galaxies, with velocity dispersions of order $2000 \mathrm{~km} \mathrm{~s}^{-1}$. This leads to a crude dependence estimate of (Mass) ${ }^{+1}$ with a large uncertainty. We estimate the stickiness to be 100 percent for the chosen crosssection, after a few spiral-down orbits. The combination would imply $\lambda=1.5$, compatible with what we obtain, allowing for large error bars. It is possible that the merger cross-section increases more steeply with mass than we have assumed here, and an exponent close to unity would allow a closer match with data. We conclude that mergers between black holes might be able to explain the entire mass distribution.

However, we have to ask whether this growth process could operate in a similar way for both nuclear star clusters and black holes. All these merger arguments may work as well for nuclear star clusters as for black holes surrounded by stars.

In this picture, the upper end of the distribution is just the maximum that can be reached given the density of galaxies and the mass of the central black holes.

\section{The transition in mass}

Why is there a minimum mass in super-massive black holes?

As we argued earlier, the available observational data and much work by Greene et al. (2008) and others show that there are very few black holes near to and below $10^{6} M_{\odot}$. There is a variety of explanations of why this is so (Portegies Zwart et al. 2004; Munyaneza \& Biermann 2006, 2005). It seems plausible to assume that the transition from massive black holes to nuclear star clusters holds a clue to solving this question. A possible transition from a nuclear star cluster to a super-massive star has been discussed by Sanders (1970), and again by Portegies Zwart et al. (2004), with the latter team arguing for a transition to a super-massive black hole (Appenzeller \& Fricke 1972b,a; Heger et al. 2003). However, the results of Appenzeller \& Fricke (1972b,a) preclude any contribution from super-massive stars near or above $10^{6} M_{\odot}$, since these stars completely explode because of an instability predicted by General Relativity, leaving no black hole behind; we therefore require no mechanism that manages to produce black holes below this cutoff. Yungelson et al. (2008) demonstrated that wind mass loss effectively competes with agglomeration, so would limit massive stars of a few hundred $M_{\odot}$ to below $100 M_{\odot}$; this implies that it would be difficult to overcome this barrier in mass. On the other hand, agglomeration is a runaway process, while stellar winds are a quasisteady process, basically limited to the Eddington luminosity; so perhaps more extreme conditions are required to achieve a serious runaway in agglomeration.

There are a number of processes that contribute to black home growth. One is the simple momentum exchange between stars on a timescale of (Chandrasekhar 1942; Spitzer 1962, 1987; Binney \& Tremaine 1987)

$\tau_{\text {grav }}=\frac{\sigma_{\star}^{3}}{8 \pi G_{N}^{2} m_{\star}^{2} n_{\star} \Lambda}$

where $\sigma_{\star}$ is the velocity dispersion of the stars in the system, which are assumed to be in virial equilibrium, $m_{\star}$ is the mass of the stars, $n_{\star}$ is the density of the stars, and $\Lambda$ is the Coulomb logarithm, typically of value 20 . For nuclear star clusters, it is difficult to understand how this process by itself would lead to a sudden transition at a specific mass, although a gravo-thermal catastrophe could in principle achieve this (Spitzer 1987); however, this process by itself implies that the objects of lower mass become a black hole and those of higher mass remain a star cluster, in contrast to observation.

On the other hand, the agglomeration of stars is governed by their collision timescale, which is

$\tau_{\mathrm{aggl}}=\frac{1}{N_{\star} n_{\star} \sigma_{\star} \Sigma_{\star}}$

where $N_{\star}$ is the total number of stars, $n_{\star}$ is the density of stars, and $\Sigma_{\star}$ is the cross-section of typical stars. We only need one star to start a runaway coalescence, and that is also why we have the factor $N_{\star}$. The question is whether either of these two processes or a combination of the two would allow a transition at a specific mass to a nuclear star cluster. McMillan \& Portegies Zwart (2007) suggest that there is a very wide mass range across which the process of agglomeration produces a wide variety of masses of intermediate mass black holes. Therefore their conclusion is that this would not lead to a relatively sharp transition.

Some have suggested (Perets \& Alexander 2008) that in the case of a binary merger of equal black holes masses a gravitational rocket effect could eject black holes from galactic centers, and one could so imagine that all lower mass black holes might form, but no longer be in galactic centers. In this case, these black holes below the transition point would exist, but be invisible. However, in Gergely \& Biermann (2009) we show that this process is unlikely to be statistically relevant. 
To summarize, if we were to accept the process of agglomeration, we ask what could modify the conclusion of previous authors that a variety of masses is formed, and reduce the mass range for the transition mass? We now consider several possibilities.

First, galaxies grow by merging, starting from some minimum size. Could this minimum size of a central black hole correspond to the minimum size of a galaxy? This is difficult to answer, even considering, that Gilmore et al. (2007) identified a minimum mass of order $5 \times 10^{7} M_{\odot}$, most of it being in dark matter. To grow a galaxy such as ours that contains a central black hole close to the low mass cut-off, would require so many merger events, that it is difficult to see that much of any connection to the minimum mass galaxy could survive in terms of some a signature, except for properties that survive in all galaxies, independent of whether they contain a black hole at their center.

Second, in a merger process we do obtain a central spike in dark matter from the merger density profile (Navarro et al. 1997; Kravtsov et al. 1998; Moore et al. 1998) $\rho_{\mathrm{dm}} \sim\left(x^{\gamma_{\mathrm{sp}}}(1+x)^{2}\right)^{-1}$ where $x=r / r_{\mathrm{c}}$, a scaled radial coordinate, and $\gamma_{\mathrm{sp}}$ is of order unity; various variants of this profile formula have been discussed (Kravtsov et al. 1998). This indicates that there is a central dark matter component of mass enclosed within $R$ of $M_{R, \mathrm{dm}} \sim R^{3-\gamma_{\mathrm{sp}}}$. Applying this first to stars alone implies a radial dependence of $n_{\star} \sim 1 / x^{\gamma_{\mathrm{sp}}}$, and velocity dispersion of $\sigma_{\star} \sim x^{\left(2-\gamma_{\mathrm{sp}}\right) / 2}$. The combination implies that $\tau_{\mathrm{aggl}} \sim x^{\left(3 \gamma_{\mathrm{sp}}-1\right) / 2}$, hence an arbitrarily short timescale of stellar agglomeration $\tau_{\text {aggl }}$ at the center of merged galaxies. This implies that all galaxies have a central super-massive black hole, not just those above a specific mass, in contradiction to observational data. However, including the process of massive star formation (e.g., Bartko et al. 2009) near the center of a galaxy might require a certain minimum amount of gaseous turn-over by star (star formation, mass ejection by winds, and explosions), and so it is conceivable that these processes define a threshold for run-away agglomeration.

Third, in a speculation on the nature of the dark matter particle's, Munyaneza \& Biermann (2006, 2005), following earlier work, suggested that a keV Fermion would naturally help to identify a mass scale from a degenerate configuration, and this mass for a dark matter particle is consistent with a few $10^{6} M_{\odot}$. This mass appears consistent with a) the low mass cutoff in the galaxy distribution found by Gilmore et al. (2007), b) the early star formation (Biermann \& Kusenko 2006; Stasielak et al. 2007; Loewenstein et al. 2009), and c) new galaxy data interpretation (de Vega \& Sanchez 2009). For some of these applications, it is important to remember that a thermal $\mathrm{keV}$ particle is already excluded as a dark matter candidate (Shaposhnikov 2007); it would have to be substantially sub-thermal.

Fourth, if supermassive stars of all masses do form, evolve, and explode, it is conceivable, that they do not form a black hole, but a naked singularity (Joshi 2007), which then due to quantum loop gravity effects disperses, leaving no remnant behind. This would have to occur only in the mass range between about 20 and $\sim 10^{6} M_{\odot}$, and neither above nor below.

Fifth, collapsing stars might undergo a phase transition to a SUSY-state (Clavelli 2006) before forming a black hole, which then also blows up all the star, leaving no remnant behind.

Finally, we need to remember, that in agglomeration of stars any massive star near to or above $10^{6} M_{\odot}$ becomes unstable and explodes, and does so on very short timescales, leaving no remnant behind (Appenzeller \& Fricke 1972b,a). Therefore, all stars reaching masses just below this limit will become a black hole, and perhaps consist of the abundances of the early universe; those very early massive stars near to but below instability threshold will also become black holes rather than completely explode. This instability defines a threshold, and makes it obvious why the initial black holes - in the agglomeration picture - cannot originate in stars of mass above about $10^{6} M_{\odot}$. If an evolving star moves up the main sequence, steadily growing in mass, it becomes ever more unstable simply due to the increasing fraction of pressure held by radiation. When it approaches instability, it might just collapse, forming a black hole just below the mass threshold. A proper evolutionary calculation is required to test this.

One could imagine, that such a dark matter core would also help to induce a runaway agglomeration process among stars as well; both run-away agglomeration and degenerate dark matter cores, or even a combination of both, seem feasible.

\section{Conclusions}

We have derived the mass function of black hole candidates in the centers of galaxies down to $3 \times 10^{6} M_{\odot}$. At high mass, it is consistent with a straight steep power law, of slope -2 in the integral mass function. Since all of the black holes that we have been able to check has been declared a black hole by more sophisticated measurements, we feel fairly confident, that almost all the black hole candidates are indeed black holes; the most likely problem with this identification is below the considered mass range.

We first conclude that this distribution function cannot possibly continue through the gap between about 30 and $10^{6} M_{\odot}$, in agreement with Greene et al. (2006), Maccarone et al. (2007) and Maccarone \& Servillat (2008). From relatively high masses, it flattens near $10^{7} M_{\odot}$, giving a contribution to the cosmological density of only

$\Omega_{\mathrm{BH}}=2 \times 10^{-6 \pm 0.40}$,

which is consistent with many other earlier estimates. As in some other analyses, our distribution flattens below $10^{8} M_{\odot}$ (Lauer et al. 2007; Benson et al. 2007).

As a corollary, we note that Frampton (2009) pointed out that black holes constitute a huge reservoir of entropy. Integrating the mass function to obtain the total entropy, we have found that the entropy is dominated by the most massive black holes, and is quite large. However, allowing for the errors in the exponent, the entropy might be logarithmically evenly distributed among all masses. Therefore, the entropy is almost unaffected by the speculation that the black hole mass function may extend to lower masses, and it is indeed curious that a logarithmically even distribution in entropy be realized in Nature. However, this implies that the summed entropy of all super-massive black holes is a reasonably well defined quantity, a new cosmological parameter, that requires an understanding.

As a second corollary, we note that the early energy input of gravitational waves from the first merging black holes approaches oddly the energy density of dark energy at a very high redshift, of order 50, possible since very massive stars may have formed at even earlier redshift, such as 80 (Biermann \& Kusenko 2006).

Second, the energy input from the growth of all these black holes is at the fraction of a percent level of the microwave background, which may mostly be lost in expansion, but some traces should remain. If some of this energy is transferred to magnetic fields, beyond what is given to intergalactic magnetic field from the large-scale gravitational potential wells, the effect on 
the scattering of ultra high energy particles could be strong. This channeling of energy, however, might present a problem. The fraction of black-hole growth energy going into this channel is constrained to be less than a few percent.

Third and last, just going to a distance of $100 \mathrm{Mpc}$, or redshift of 0.025 , and going to $10^{7} M_{\odot}$ our results give $2.4 \times 10^{4}$ black holes, or on the sky 0.6 per every square degree. There is some evidence that every super-massive black hole is active at an observable level in radio emission (Nagar et al. 2005). Radio emission implies non-thermal particles, and so the speculation, that each and everyone might contribute also to ultra high energy cosmic rays, is not immediately discountable. More specific models, with testable predictions, are required to investigate this possibility.

Acknowledgements. Useful and inspiring discussions with R. Beck, J. Becker, S. Britzen, L. Clavelli, T. Enßlin, L. Gergely, Gopal-Krishna, B. Harms, E. van den Heuvel, J. Irwin, P. Joshi, H. Kang, T. Kneiske, P.P. Kronberg, N. Langer, T. Maccarone, S. Portegies-Zwart, J. Rachen, D. Ryu, N. Sanchez, T. Stanev, H. de Vega, and P. Wiita are gratefully acknowledged. We thank the anonymous referee for very useful corrections and comments. Work with PLB was supported by contract AUGER 05 CU 5PD 1/2 via DESY/BMB and by VIHKOS via FZ Karlsruhe; by Erasmus/Sokrates EU-contracts with the universities in Bucharest, Cluj-Napoca, Budapest, Szeged, Cracow, and Ljubljana; by the DFG, the DAAD and the Humboldt Foundation; and by research foundations in Japan, Korea, China, Australia, India, Europe, the USA and Brazil. L.I.C. is partially supported by CNCSIS Contract 539/2009 and CNMP Contract 82077/2008. L.I.C. wishes to express his thanks to the MPIfR for support when finishing this project. This research has made use of the NASA/IPAC Extragalactic Database (NED) which is operated by the Jet Propulsion Laboratory, California Institute of Technology, under contract with the National Aeronautics and Space Administration. This research also made use of the ViZier system at the Centre de Donneés astronomiques de Strasbourg (CDS) (Ochsenbein et al. 2000).

\section{References}

Abramowicz, M. A., Becker, J. K., Biermann, P. L., et al. 2009, ApJ, 705, 659 Abubekerov, M. K., Antokhina, E. A., Bogomazov, A. I., \& Cherepashchuk, A. M. 2009, Astron. Rep., 53, 232

Agol, E., \& Kamionkowski, M. 2002, MNRAS, 334, 553

Aller, M. C., \& Richstone, D. O. 2007, ApJ, 665, 120

Appenzeller, I., \& Fricke, K. 1972a, A\&A, 18, 10

Appenzeller, I., \& Fricke, K. 1972b, A\&A, 21, 285

Barth, A. J., Ho, L. C., Rutledge, R. E., \& Sargent, W. L. W. 2004, ApJ, 607, 90

Barth, A. J., Greene, J. E., \& Ho, L. C. 2005, ApJ, 619, L151

Barth, A. J., Bentz, M. C., Greene, J. E., \& Ho, L. C. 2008a, ApJ, 683, L119

Barth, A. J., Greene, J. E., \& Ho, L. C. 2008b, AJ, 136, 1179

Barth, A. J., Strigari, L. E., Bentz, M. C., Greene, J. E., \& Ho, L. C. 2009, ApJ, 690, 1031

Bartko, H., Martins, F., Fritz, T. K., et al. 2009, ApJ, 697, 1741

Benson, A. J., Džanović, D., Frenk, C. S., \& Sharples, R. 2007, MNRAS, 379, 841

Biermann, P. L., \& Frampton, P. H. 2006, Phys. Lett. B, 634, 125

Biermann, P. L., \& Kusenko, A. 2006, Phys. Rev. Lett., 96, 091301

Binney, J., \& Tremaine, S. 1987, Galactic dynamics

Blandford, R. D., \& Znajek, R. L. 1977, MNRAS, 179, 433

Breitschwerdt, D. 2008, Nature, 452, 826

Caramete, L. I., \& Biermann, P. L. 2010, in preparation

Casella, P., Ponti, G., Patruno, A., et al. 2008, MNRAS, 387, 1707

Chandrasekhar, S. 1942, Principles of stellar dynamics

Clark, J. S., Goodwin, S. P., Crowther, P. A., et al. 2002, A\&A, 392, 909

Clavelli, L. 2006, High Energy Density Physics, 2, 97

Côté, P., Piatek, S., Ferrarese, L., et al. 2006, ApJS, 165, 57

de Vega, H. J., \& Sanchez, N. G. 2009, preprint [arXiv:0907.0006]
Enßlin, T. A., Wang, Y., Nath, B. B., \& Biermann, P. L. 1998, A\&A, 333, L47 Everett, J. E., Zweibel, E. G., Benjamin, R. A., et al. 2008, ApJ, 674, 258 Faber, S. M., Tremaine, S., Ajhar, E. A., et al. 1997, AJ, 114, 1771

Falcke, H., \& Biermann, P. L. 1995, A\&A, 293, 665

Farrell, S. A., Webb, N. A., Barret, D., Godet, O., \& Rodrigues, J. M. 2009, Nature, 460, 73

Frampton, P. H. 2009, preprint [arXiv:0905.2535]

Gebhardt, K., Rich, R. M., \& Ho, L. C. 2005, ApJ, 634, 1093

Gergely, L. Á., \& Biermann, P. L. 2009, ApJ, 697, 1621

Gilmore, G., Wilkinson, M. I., Wyse, R. F. G., et al. 2007, ApJ, 663, 948

Gopal-Krishna, \& Wiita, P. J. 2001, ApJ, 560, L115

Greene, J. E., \& Ho, L. C. 2007a, ApJ, 670, 92

Greene, J. E., \& Ho, L. C. 2007b, in The Central Engine of Active Galactic

Nuclei, ed. L. C. Ho, \& J.-W. Wang, ASP Conf. Ser., 373, 33

Greene, J. E., Barth, A. J., \& Ho, L. C. 2006, New Astron. Rev., 50, 739

Greene, J. E., Ho, L. C., \& Barth, A. J. 2008, ApJ, 688, 159

Häring, N., \& Rix, H.-W. 2004, ApJ, 604, L89

Heger, A., Fryer, C. L., Woosley, S. E., Langer, N., \& Hartmann, D. H. 2003, ApJ, 591, 288

Irwin, J., Bregman, J., \& Brink, T. 2009, in BAAS, 41, B, 310

Joshi, P. S. 2007, Pramana, 69, 119

Komatsu, E., Dunkley, J., Nolta, M. R., et al. 2009, ApJS, 180, 330

Körding, E., Falcke, H., \& Markoff, S. 2002, A\&A, 382, L13

Kravtsov, A. V., Klypin, A. A., Bullock, J. S., \& Primack, J. R. 1998, ApJ, 502, 48

Kronberg, P. P., Dufton, Q. W., Li, H., \& Colgate, S. A. 2001, ApJ, 560, 178

Lauer, T. R., Faber, S. M., Richstone, D., et al. 2007, ApJ, 662, 808

Loewenstein, M., Kusenko, A., \& Biermann, P. L. 2009, ApJ, 700, 426

Lüst, R. 1952, Zeitschr. f. Naturf., 87

Maccarone, T., \& Koerding, E. 2006, Astron. Geophys., 47, 060000

Maccarone, T. J., \& Servillat, M. 2008, MNRAS, 389, 379

Maccarone, T. J., Kundu, A., Zepf, S. E., \& Rhode, K. L. 2007, Nature, 445, 183

Magorrian, J., Tremaine, S., Richstone, D., et al. 1998, AJ, 115, 2285

Marochnik, L., Usikov, D., \& Vereshkov, G. 2008, preprint [arXiv:0811.4484]

McMillan, S. L. W., \& Portegies Zwart, S. F. 2007, in Massive Stars in Interactive Binaries, ed. N. St.-Louis, \& A. F. J. Moffat, ASP Conf. Ser., 367, 697

Moore, B., Governato, F., Quinn, T., Stadel, J., \& Lake, G. 1998, ApJ, 499, L5

Munyaneza, F., \& Biermann, P. L. 2005, A\&A, 436, 805

Munyaneza, F., \& Biermann, P. L. 2006, A\&A, 458, L9

Nagar, N. M., Falcke, H., \& Wilson, A. S. 2005, A\&A, 435, 521

Navarro, J. F., Frenk, C. S., \& White, S. D. M. 1997, ApJ, 490, 493

Novikov, I. D., \& Thorne, K. S. 1973, in Black Holes (Les Astres Occlus), 343

Orosz, J. A., McClintock, J. E., Narayan, R., et al. 2007, Nature, 449, 872

Patruno, A., Portegies Zwart, S., Dewi, J., \& Hopman, C. 2006, MNRAS, 370, L6

Perets, H. B., \& Alexander, T. 2008, ApJ, 677, 146

Portegies Zwart, S. F., Baumgardt, H., Hut, P., Makino, J., \& McMillan, S. L. W. 2004, Nature, 428, 724

Ryu, D., Kang, H., Cho, J., \& Das, S. 2008, Science, 320, 909

Sanders, R. H. 1970, ApJ, 162, 791

Schechter, P. 1976, ApJ, 203, 297

Shakura, N. I., \& Sunyaev, R. A. 1973, A\&A, 24, 337

Shaposhnikov, M. 2007, ArXiv Astrophysics e-prints

Silk, J., \& Takahashi, T. 1979, ApJ, 229, 242

Silk, J., \& Rees, M. J. 1998, A\&A, 331, L1

Silverman, J. M., \& Filippenko, A. V. 2008, ApJ, 678, L17

Skrutskie, M. F., Cutri, R. M., Stiening, R., et al. 2006, AJ, 131, 1163

Spitzer, L. 1962, Physics of Fully Ionized Gases

Spitzer, L. 1987, Dynamical evolution of globular clusters

Stasielak, J., Biermann, P. L., \& Kusenko, A. 2007, ApJ, 654, 290

Tremaine, S., Gebhardt, K., Bender, R., et al. 2002, ApJ, 574, 740

Trudolyubov, S., \& Priedhorsky, W. 2004, ApJ, 616, 821

Ulvestad, J. S., Greene, J. E., \& Ho, L. C. 2007, ApJ, 661, L151

van der Wel, A., Franx, M., van Dokkum, P. G., et al. 2006, ApJ, 636, L21

van Kerkwijk, M. H., Geballe, T. R., King, D. L., van der Klis, M., \& van Paradijs, J. 1996, A\&A, 314, 521

Wang, Y., \& Biermann, P. L. 1998, A\&A, 334, 87

Yungelson, L. R., van den Heuvel, E. P. J., Vink, J. S., Portegies Zwart, S. F., \& de Koter, A. 2008, A\&A, 477, 223 